\section{Drs. Mori and Cho reply}

\section{To the Editor:}

We thank Dr. Georgiadis and colleagues for their comments on our study ${ }^{1}$. We are pleased that our findings have attracted the interest of readers, and we offer the following response.

First, Georgiadis, et al state that we reported no significant differences in HRCT findings between patients with early RA and those with longstanding RA, but we think that this is a misinterpretation. We performed HRCT on a total of 126 patients with either early RA $(n=65)$ or longstanding RA $(n=61)$. Abnormalities on HRCT imaging were categorized, and the frequency of each finding and its extent in the lungs were compared between the 2 patient populations. We found that the frequency of interstitial abnormalities such as ground-glass attenuation, reticulation, honeycombing, and consolidation was not significantly different between the 2 groups. Yet it is important to recall that, in contrast, there were significant differences in the frequencies of parenchymal micronodules and bronchial wall thickening, both of which are indicative of small-airway diseases; specifically, these conditions were more prominently observed in the patients with longstanding RA, which tendency apparently contributed to the higher incidence of the bronchiolitis pattern in this group. We therefore concluded that the incidence of bronchiolar abnormalities is associated with the duration of RA. The longstanding RA group appeared to be predisposed to complications of small-airway disease. These findings do not contradict the claim that rheumatoid lung disease occurs more frequently in patients with longstanding $\mathrm{RA}^{2}$. A high prevalence of airway abnormalities was also observed in several previous HRCT studies on patient populations with relatively long mean disease durations ${ }^{3-5}$.

As we mentioned in our article, the $\mathrm{C}$-reactive protein value, a measure of disease activity, was significantly higher in the patients with longstanding RA, but other disease activity measures, including the DAS-28 and the erythrocyte sedimentation rate, were measured at similar levels in the 2 patient groups. Therefore, it is not clear whether the increased incidence of small-airway abnormalities may reflect high levels of disease activity. Based on the extent of their joint and bone damage, all patients in the early RA group were assigned to either Steinbrocker stage I (48 of 65 patients, $74 \%$ ) or stage II (17 patients, $26 \%$ ). In the longstanding RA group, all patients except one were assigned to either Steinbrocker stage III (26 of 61, $43 \%$ ) or IV (34 of $61,56 \%$ ). Thus the extent of joint and bone damage may be one factor associated with increased risk of development of bronchiolitis. In addition, a previous study cited by Georgiadis, et al has shown that early disability (Steinbrocker stage III-IV) is a risk factor for extraarticular $\mathrm{RA}^{6}$. In the 2 patient groups in our study, no extraarticular manifestations other than pulmonary abnormalities were observed.

Smoking has been reported to increase the risk of developing pulmonary diseases. In our study, the numbers of current and former smokers were small (13 patients in the early RA group and 8 in the longstanding RA group), and only 3 of these were diagnosed as having either the usual interstitial pneumonia (UIP) pattern (1 case) or the nonspecific interstitial pneumonia (NSIP) pattern ( 2 cases). We have obtained no evidence that smoking has any effect on the incidence of pulmonary abnormalities on HRCT scans.

Another factor that has been suggested to predispose RA patients to the development of pulmonary abnormalities is the previous use of DMARD. We therefore excluded from our study patients who had received MTX and anti-tumor necrosis factor- $\alpha$ (TNF- $\alpha$ ) agents. As for the other DMARD, 11 patients in the early RA group had received therapy with either bucillamine, an analog of D-penicillamine (6 patients, $9 \%$ ), or sulfasalazine (5 patients, $8 \%$ ) for several months, and only 1 case among these was diagnosed with the NSIP pattern. In the longstanding RA group, most patients had used D-penicillamine and/or gold salts. We cannot entirely exclude the possibility that the use of these is associated with an increased risk of lung disease in this patient population, and it is unclear whether such an association, if it exists, is due to drug-related events or simply reflects the severity and/or duration of the underlying disease.
We appreciate the suggestions by Georgiadis, et al that our study would be further enhanced by a matched control group undergoing similar pulmonary investigations. An age- and sex-matched healthy control group would provide data on the background prevalence of pulmonary changes in the general population. Yet we hesitate to perform HRCT examinations on healthy individuals, because it is generally accepted that the use of diagnostic radiographs such as HRCT may increase a person's risk of developing cancer ${ }^{7}$. This may be why most studies on pulmonary abnormalities in RA perform HRCT examinations only on patients who are already suspected of having lung diseases. In our hospital, an HRCT examination is performed on all patients with RA unless they refuse it, since it is essential to carefully examine pulmonary conditions before introducing MTX and/or anti-TNF- $\alpha$ therapy. This policy made it possible to characterize pulmonary abnormalities in unselected RA patients. We did administer PFT to the healthy control group, and we found that the mean values of $\mathrm{FEV}_{1}$ /forced vital capacity ratio and $\mathrm{FEV}_{25-75}$ were significantly lower in patients with the bronchiolitis pattern, and that the mean DLCO was significantly decreased in patients with the NSIP or UIP pattern.

Georgiadis and colleagues have shown that lung abnormalities on HRCT scans are frequently observed in patients with early RA who do not have respiratory symptoms, even when plain chest radiograph and PFT are normal, and that ground-glass opacities are seen exclusively in patients with RA and not in healthy control subjects ${ }^{8}$. These findings are of interest since they may suggest that the presence of this type of pulmonary HRCT abnormality is related to the occurrence of rheumatoid lung disease. Similarly, we also indicated that interstitial abnormalities are frequently observed in patients with RA, even in the early stages, although most of these patients have no respiratory symptoms. Further, we found in our study that ground-glass attenuation, the finding associated with the NSIP pattern, was the most prominent interstitial abnormality in both early and longstanding RA groups. Gabbay, et al have also shown that interstitial disease in early RA is a frequent finding on HRCT, even in asymptomatic patients, and that one of the most common interstitial abnormalities is ground-glass attenuation ${ }^{9}$. Longitudinal studies will be required to determine whether the appearance of interstitial abnormalities in the early stages of RA predicts the subsequent development of respiratory diseases or is simply a benign finding.

SHUNSUKE MORI, MD, PhD, Clinical Research Center for Rheumatic Disease and Department of Rheumatology; ISAMU CHO, MD, PhD, Clinical Research Center for Rheumatic Disease and Division of Respiratory Medicine, Department of Medicine, Kumamoto Saishunsou National Hospital, Kohshi, Japan. Address reprint requests to Dr. S. Mori, Clinical Research Center for Rheumatic Disease and Department of Rheumatology, Kumamoto Saishunsou National Hospital, 2659 Suya, Kohshi, Kumamoto 861-1196, Japan.

E-mail: moris@saisyunsou1.hosp.go.jp

\section{REFERENCES}

1. Mori S, Cho I, Koga Y, Sugimoto M. Comparison of pulmonary abnormalities on high-resolution computed tomography in patients with early versus longstanding rheumatoid arthritis. J Rheumatol 2008;35:1513-21.

2. Anaya JM, Diethelm L, Ortiz LA, et al. Pulmonary involvement in rheumatoid arthritis. Semin Arthritis Rheum 1995;24:242-54.

3. Terasaki H, Fujimoto K, Hayabuchi N, Ogoh Y, Fukuda T, Muller NL. Respiratory symptoms in rheumatoid arthritis: Relation between high resolution CT findings and functional impairment. Radiat Med 2004;22:179-85.

4. Cortet B, Perez T, Roux N, et al. Pulmonary function tests and high resolution computed tomography of the lungs in patients with rheumatoid arthritis. Ann Rheum Dis 1997;56:596-600.

5. Perez T, Remy-Jardin M, Cortet B. Airways involvement in rheumatoid arthritis: Clinical, functional, and HRCT findings. Am 
J Respir Crit Care Med 1998;157:1658-65.

6. Turesson C, O'Fallon WM, Crowson CS, Gabriel SE, Matteson EL. Extra-articular disease manifestations in rheumatoid arthritis: Incidence trends and risk factors over 46 years. Ann Rheum Dis 2003;62:722-7.

7. Berrington de Gonzalez A, Darby S. Risk of cancer from diagnostic X-rays: Estimates for the UK and 14 other countries. Lancet 2004:363:345-51.

8. Metafratzi ZM, Georgiadis AN, Ioannidou CV, et al. Pulmonary involvement in patients with early rheumatoid arthritis. Scand J Rheumatol 2007;36:338-44.

9. Gabbay E, Tarala R, Will R, et al. Interstitial lung disease in recent onset rheumatoid arthritis. Am J Respir Crit Care Med 1997;156:528-35.

J Rheumatol 2009;36:2; doi:10.3899/jrheum.081003 